\title{
Formation of Ag Fine Crystal by Heat Decomposition of Fatty Acid Ag Salt
}

\author{
Kazuhiro HONDA \\ Department of Applied Chemistry, Kanagawa Institute of Technology ; \\ Atsugi-shi 243-0292, Japan \\ Fax:81-46-242-8760,E-mail:honda@chem.kanagawa-it.ac.jp
}

We examined recovery of silver ion using fatty acid sodium (Soap) and heat treatment of the formed fatty acid silver (Scum) under the oxygen atmosphere. Fatty acid sodium reacted with the silver ion, and the fatty acid silver was formed in the high yield. The formed fatty acid silver was thermally unstable, and it decomposed by the heating of low temperature of about $130{ }^{\circ} \mathrm{C}$ under the oxygen atmosphere, and the silver was formed.

The development of the low energy type of recycling system is greatly expected, because the silver can be by far formed in low temperature than the melting point of bulk silver.

Key words: fatty acid sodium(Soap), fatty acid silver (Scum), heat decomposition, Ag fine particle

\section{INTRODUCTION}

Recently, the challenge to the environmental problem became popular, and the interest would be collected in the water purification technology.

It is important to recover the discharged metal ion as a result of the industrial activity from the viewpoint of environmental preservation and effective utilization of resources.

The silver which is valuable metal is included in the waste water discharged from galvanizers industry, etc., and the emission regulation becomes recently severe more and more.

The low-cost and efficient recovery technique of silver ion in waste water is required. At present, the following exist as a silver ion recovery method: Ion-exchange column method ${ }^{[1]}$, adsorption column method, membrane separation $\operatorname{method}^{[2]}$, sedimentation method, liquid-liquid extraction method ${ }^{[2]}$.

Especially, liquid-liquid extraction method and adsorption column method by the chelating resin using the polymeric adsorbent are greatly noticed as an advanced waste water treatment technology. However, the adsorption capacity for these extraction methods is small, and the after treatment of the large-scale drainage is require in order to use the noxious organic solvent (toluene, etc.,).

The separation from the resin of adsorbed metal ion is not always easy for the extracting method by the polymeric resin adsorbent which modified chelating resin chemically.

The extraction of noble metal ion will finally incinerate the resin, and there is a problem for the environment that the incineration product in which the after treatment of tar and coke is troublesome remains. And, it is a problem that the adsorbent with the chelating resin is expensive economically.

Such fact becomes a background, precipitation 
separation method removed as a practical processing as an insoluble precipitation becomes a general.

The purpose of this study is the establishment of silver ion recovery technique of the environmental harmony type that the toxic substance is not discharged and energy-saving high yield silver recovery technique without completely using toxic substance in the recovery process.

Concretely, the recovery of silver ion by the formation of the insoluble fatty acid silver ( scum ) using the biodegradable fatty acid sodium and the formation of Ag crystal by thermal decomposition under the oxygen atmosphere was examined.

\section{EXPERIMENTAL}

The marketing reagent $\mathrm{AgNO}_{3}$ (WAKO Co.,Ltd, $>99.8 \%$ ) was used as it is. Fatty acid sodium used the general marketing pure soap that the silicate was produced by using for alkali medicine. Also the water content of soap was analyzed by the moisture percentage measurement (AND Co.Ltd; MX-50), and it was considered for the preparation of the concentration of soapsuds solution.

For the beginning, $200 \mathrm{mM}$ silver nitrate aqueous solution and soapsuds solutions of largest concentration of 4 mass $\%$ were prepared to $50 \mathrm{ml}$, respectively. The reaction was carried out for $10 \mathrm{~min}$, after the all soapsuds solution was quickly put into the silver nitrate aqueous solution stirred in the $40{ }^{\circ} \mathrm{C}$ thermostatic bath.

The sample after the reaction carried out the filtration. The concentration of silver ion which remained in separated supernatant solution was analyzed by a Mohr method.

Formed fatty acid silver measured the mass change by the sequential heating, after it dried at room temperature in the darkroom. The sequential heating from $30{ }^{\circ} \mathrm{C}$ to $260{ }^{\circ} \mathrm{C}$ heated $10{ }^{\circ} \mathrm{C}$ at 24 hours by in the drying oven.

Production of the Ag crystal after the heat treatment of the fatty acid silver was confirmed from the measurement of X-ray diffraction (XRD) pattern by using the Rigaku: RAD- II VC diffractmeter ( $\mathrm{CuK} \alpha$ radiation).

And, the shape of formed silver crystal was observed by a scanning electron microscope (SEM; JEOL; JNN-LA300).

\section{RESULTS AND DISCUSSION}

For the initial concentration of $100 \mathrm{mM}$ silver ion, the result of the removal concentration of the silver ion by the change of the soap concentration from 0.1 mass $\%$ to 2.0 mass\% is shown in Fig .1.

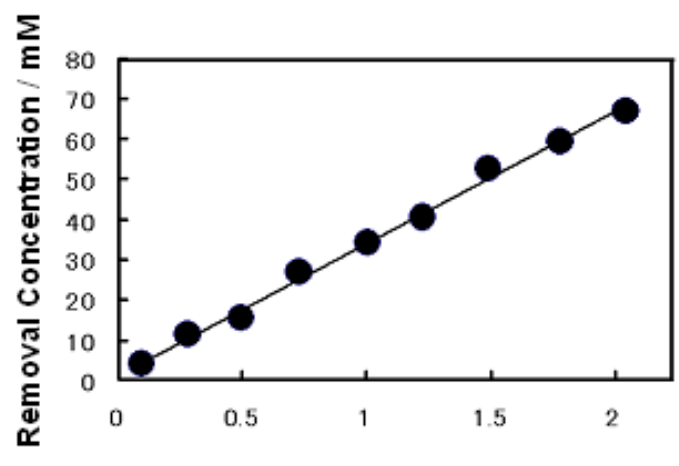

Soap concentration /mass \%

Fig.1 Removal concentration of silver ion by changing the soap concentration. Initial concentration of silver ion and soap concentration are $100 \mathrm{mM}$ and 0.1 mass\% to 2.0 mass\%, respectively.

It was shown that the relation between removal concentration of the silver ion and added soap concentration was the linear relation. It was enabled that $70 \mathrm{mM}$ concentration of silver ion was removed 
from aqueous solution by adding the 2.0 mass $\%$ concentration of soap .

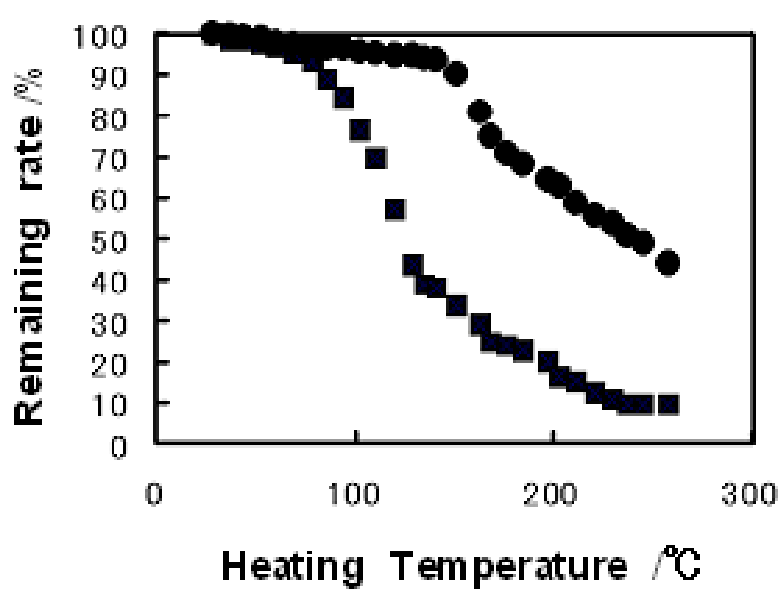

Fig.2 Changes of the weight by the sequential heating formed fatty acid silver ( $\square$ ) and Soap (O). Vertical axis shows the change of remaining rate with the sequential heating, and the rate at the heating start of $30{ }^{\circ} \mathrm{C}$ is $100 \%$.

The changes of the weight by the sequential heating from $30{ }^{\circ} \mathrm{C}$ to $260{ }^{\circ} \mathrm{C}$ of formed fatty acid silver and fatty acid sodium (Soap) are shown in Fig.2. The change of increasing temperature is $10{ }^{\circ} \mathrm{C}$ at 24 hours.

Formed fatty acid silver showed the gentle mass decrease with the heating start, and the rapid mass decrease in the vicinity of $80{ }^{\circ} \mathrm{C}$ was shown.

In the vicinity of $130{ }^{\circ} \mathrm{C}$, the decreasing rate became gentle, and the change of decreasing rate approached to zero in the $250{ }^{\circ} \mathrm{C}$ vicinity.

It was observed that fatty acid silver changed to the melt with the viscosity by the heating in the vicinity of $130{ }^{\circ} \mathrm{C}$. The melt started the decomposition over $200{ }^{\circ} \mathrm{C}$, and it changed to the yellow solid. This color change strongly indicates the formation of silver ultrafine particle with the size of $5 \mathrm{~nm}$ or less $^{[3]}$. The color change finally showed yellow green in the $250{ }^{\circ} \mathrm{C}$
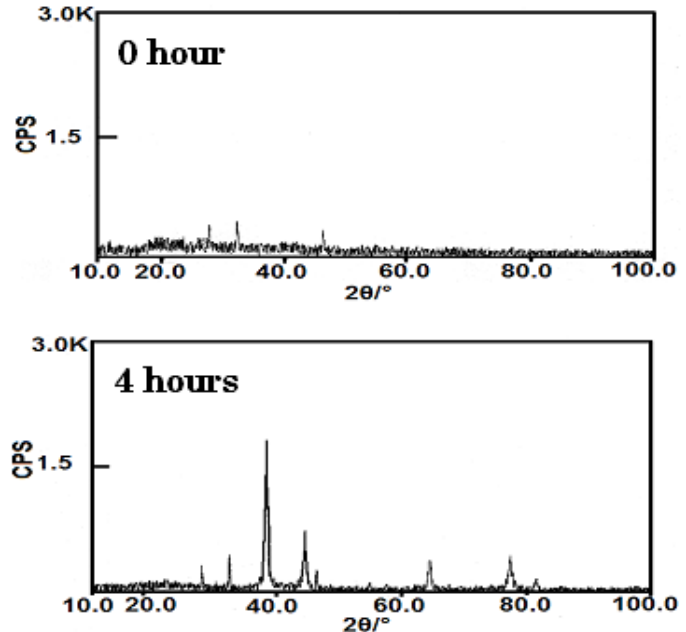

Fig.3 XRD pattern of formed fatty acid silver

( 0 hour) and after the heating at $250{ }^{\circ} \mathrm{C}$

(4 hours) sample.

vicinity.

On the other hands, it became clear that decomposition behavior of the fatty acid sodium was shifting to the high temperature of about $100{ }^{\circ} \mathrm{C}$, in comparison with the result of fatty acid silver .

The X-ray diffraction (XRD) pattern of the formed fatty acid silver ( 0 hour) and after the heating at $250{ }^{\circ} \mathrm{C}$ (4 hours) sample are shown in Fig.3.

It is not possible that XRD pattern of formed fatty acid silver confirms the clear diffraction peak.

On the other hand, it is possible that XRD pattern of the decomposition product by heating of the fatty acid silver confirms the clear diffraction peak.

The diffraction pattern shows clear peaks of fcc-Ag. The decomposition product by heating of the fatty acid silver were crystalline fcc- $\mathrm{Ag}^{[4]}$. Because $\mathrm{Ag}$ was formed under the conditions in which a reducing agent does not coexist, it is suggested that the fatty acid silver was reduced by the electron transfer under the dissociation process.

A SEM image of the aggregate of formed fatty acid 
silver and after the heating sample (1 hour, 2 hours , 4 hours ) are shown in Fig.4.

The fatty acid silver before the heating can confirm the structure of the ruggedness of about $500 \mathrm{~nm}$. In addition, it is possible that the surface of the ruggedness has the smoothness of the nano size.

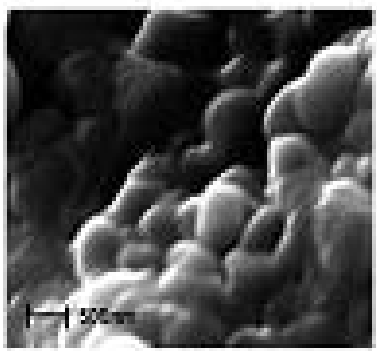

(A)

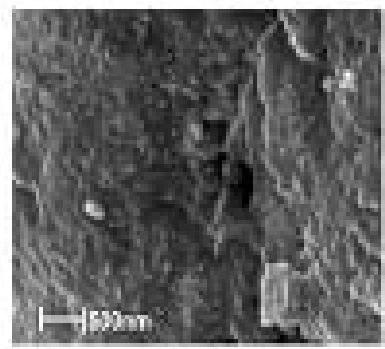

(C)

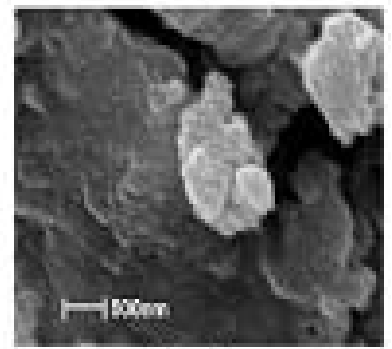

(B)

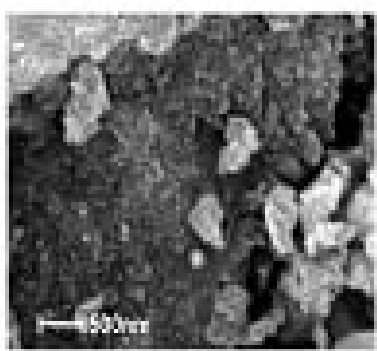

(D)
Fig.4 SEM image of formed fatty acid silver and after the heating sample; (A) 0 hour , (B) 1 hour, (C) 2 hours, (D) 4 hours . The heating temperature is $250{ }^{\circ} \mathrm{C}$. The photographing magnification are 20000 times.

By heating of $250{ }^{\circ} \mathrm{C}$ for 1 hour, it can be confirmed that the structure of the ruggedness of the fatty acid silver disappeared. By heating of 1 hour, it was able to confirm the formation of the tabular structure with the ruggedness of the nano size.

By the heating of 2 hours, the formation of the dome of the several ten nm size were able to be confirmed on the surface of the tabular structure, and they increased the number by the heating of 4 hours, and further it cleared.

From the results of SEM observation and XRD pattern, silver crystal which was produced by the heating seems to be an aggregate of silver ultrafine particles which agglutinates in the heating process.

\section{CONCLUSIONS}

The fatty acid sodium (Soap) is chemical substance which is gentle in the environment with the biodegradability.

Fatty acid sodium (Soap) formed the fatty acid silver (Scum) with the silver ion in high yield.

Fatty acid silver which was produced under the oxygen atmosphere was thermally unstable, and it decomposed in the vicinity of $130{ }^{\circ} \mathrm{C}$, the silver crystallite was formed.

The development of the low energy type of recycling system is greatly expected, because the silver can be by far formed in low temperature than the melting point of bulk silver.

\section{References.}

[1]J. B. Brower, R. L. Ryan and M. Pazirandeh, Environ. Sci. Techol, 31, 2910 (1997).

[2]L. L. Tavlarides, J. H. Baw and K. Lee, Sepa. Sci. Tech, 22, 581(1987).

[3] H. Nagasawa, M. Maruyama, T. Komatsu, S. Isoda and T. Kobayashi, physica status solidi (a) 191. p67(2002).

[4]Powder Diffraction File, Card No. 4-0783.

International Centre for Diffraction Date, Netwok

Square PA, (1996).

(Received May 14, 2010; Accepted September 6, 2010) 